\title{
Novel FGFR inhibitor ponatinib suppresses the growth of non-small cell lung cancer cells overexpressing FGFR1
}

\author{
MINGQIANG REN ${ }^{1}$, MEI HONG ${ }^{1}$, GENTAO LIU $^{5}$, HONGJIN WANG $^{5}$, VIJAY PATEL ${ }^{3}$, \\ PAUL BIDDINGER $^{4}$, JEANE SILVA $^{1}$, JOHN COWELL ${ }^{1}$ and ZHONGLIN HAO ${ }^{1,2}$ \\ ${ }^{1}$ Cancer Center, ${ }^{2}$ Division of Hematology and Oncology, Department of Medicine; and Departments of \\ ${ }^{3}$ Cardiothoracic Surgery, ${ }^{4}$ Pathology, Medical College of Georgia, Georgia Health Sciences University, \\ Augusta, GA 30912, USA; ${ }^{5}$ Center for Translational Research, Shanghai Pulmonary Hospital, \\ Tongji University School of Medicine, Shanghai 200433, P.R. China
}

Received December 12, 2012; Accepted January 10, 2013

DOI: 10.3892/or.2013.2386

\begin{abstract}
Lung cancer is still the leading cause of cancerrelated deaths worldwide. Identifying new oncogenic drivers and developing efficient inhibitors through molecular targeting approaches are crucial for improving therapies. The aim of this study was to investigate whether targeting fibroblast growth factor receptor 1 (FGFR1) with ponatinib inhibits the cell growth in both established and primary lung cancer cells overexpressing FGFR1. Eighty-eight non-small cell lung cancer (NSCLC) and paired normal tissue specimens were analyzed by real-time RT-PCR for FGFR1 gene expression. We identified four cell lines and two newly established primary lung cancer cultures that showed high FGFR1 expression levels, and evaluated the effect of the novel FGFR1 inhibitor ponatinib on cell growth. Approximately 50\% (30 out of 59) NSCLC specimens expressed FGFR1 >2-fold compared with their adjacent normal counterparts using quantitative RT-PCR. Ponatinib treatment of established NSCLC cell lines expressing higher levels of FGFR1 resulted in marked cell growth inhibition and suppression of clonogenicity. This growth inhibition was associated with inactivation of FGFR1 and its downstream targets. FGFR1 knockdown by shRNA achieved similar results when compared to treatment with ponatinib. Furthermore, ponatinib was able to significantly inhibit the growth of primary lung cancer cultures in vitro. Our data indicate that pharmacological inhibition of FGFR1 kinase activity with ponatinib may be effective for the treatment of lung cancer patients whose tumors overexpress FGFR1.
\end{abstract}

Correspondence to: Dr Zhonglin Hao, Division of Hematology and Oncology, Department of Medicine, Thoracic Oncology Program, Georgia Health Sciences University, 1120 15th Street, Augusta, GA 30912, USA

E-mail: zhao@georgiahealth.edu

Key words: fibroblast growth factor receptor 1, non-small cell lung cancer, ponatinib

\section{Introduction}

Lung cancer is the leading cause of cancer-related deaths for both males and famales in the US and worldwide. An estimated 160,340 deaths, accounting for $\sim 28 \%$ of all cancer deaths, were estimated to occur in the US in 2012 (American Cancer Society. Cancer Facts \& Figures 2012. Atlanta: American Cancer Society; 2012). Based on histological characteristics, lung cancer is classified clinically as small-cell (SCLC) (15\%) or non-small cell lung cancer (NSCLC) (85\%) (1). Adenocarcinoma and squamous cell carcinoma (SQC) are the most common histological subtypes accounting for $\sim 50$ and $30 \%$ of NSCLC cases, respectively (2). During the past decade, several oncogenic driver mutations including EGFR, EML4-ALK, ROS and RET have been identified in lung cancer. Molecularly targeted therapies against the EGFR and ALK mutations are showing success (3). Despite these improvements in molecular diagnosis and targeted therapies, the 5-year overall survival rate for all stages of lung cancer is only 16\% (American Cancer Society. Cancer Facts \& Figures 2012. Atlanta: American Cancer Society; 2012). The lack of substantial improvement in cancer mortality is multifactorial. In part, this is because the proportion of patients that carry the EGFR mutation or ALK fusion gene are only 12 and $4 \%$ of all adenocarcinoma cases, and the EGFR mutation is concentrated in female non-smokers. Clearly, discovering novel oncogenic drivers in NSCLC is paramount for improving current therapy. Recently, the fibroblast growth factor receptor 1 (FGFR1) was identified to be frequently amplified in squamous cell lung cancer ( $>20 \%$ of cases) $(4,5)$ as well as in small-cell lung cancer (6\% of cases) (6). However, it is still unknown whether these cells are completely addicted to FGFR1 signaling and whether pharmacologically targeting FGFR1 is effective in inhibiting cancer growth.

FGFR1 is a transmembrane tyrosine kinase receptor that plays an important role in cell growth, differentiation and survival among other functions (7). FGFR1 activation leads to downstream signaling via the PI3K-AKT, RAS-MEK-MAPK, STAT, PLC $\gamma$, as well as the Src signaling pathway $(8,9)$. Recently, ponatinib, also known as AP24534, has been proven 
effective against FGFR1 kinase (10). Indeed, it proved to be a potent inhibitor of leukemogenesis induced by the FGFR1 fusion kinases $(11,12)$. However, it is still not clear whether ponatinib potently inhibits NSCLC cell growth or blocks progression of tumors with FGFR1 overexpression. In the present study, we found that approximately half (30 out of 59) of all NSCLC cases showed a $>2$-fold increase in transcriptional activity of FGFR1 compared with their adjacent 'normal' tissue counterparts by using quantitative RT-PCR, indicating that not only genomic amplification of FGFR1 but also transcriptional overexpression of FGFR1 is a relative common molecular signature in NSCLC. We further demonstrated that ponatinib can effectively and specifically suppress cell growth in lung cancer cell lines as well as in primary lung cancer cell cultures with overexpression of FGFR1. This growth inhibition by ponatinib is associated with inactivation of FGFR1 and its downstream targets. Our data suggest that ponatinib is a potent drug for targeted therapy in lung cancer patients with amplification of FGFR1, and provide a rationale for further evaluation preclinically and clinically.

\section{Materials and methods}

Patient samples. Human tissue samples were obtained from the biorepositories of the Georgia Health Sciences University Cancer Center and Shanghai Pulmonary Hospital that collect anonymized samples from cancer and non-cancer patients for research purposes following protocols approved by the Georgia Health Sciences University Human Assurance Committee (GHSUHAC) and the Tongjin University Institution Review Board (TUIRB). All patients signed written consents documenting donation of their tissue for research purposes prior to tissue deposition. The 88 tumor samples together with matched adjacent normal specimens were from lung cancer patients who had undergone resection of lung cancer at the Shanghai Pulmonary Hospital (Tongji University School of Medicine, Shanghai, China) in 2009. All tumors and their paired normal tissue samples were snap frozen and stored at $-80^{\circ} \mathrm{C}$ until assayed after histological confirmation. Human lung cancer tissue samples used for isolation of primary lung cancer cell cultures were obtained from the Georgia Health Sciences University, Cancer Center Tumor Tissue and Serum Repository after de-identification.

Published microarray data set. Global gene expression profiles of lung cancer cell lines $(n=89)$ were downloaded and isolated from published microarray data sets from the cancer cell line project (950 assays, E-MTAB-37; European Bioinformatics Institute). The global gene expression levels from these lung cancer cell lines compared with the normal Wi38 human fetal lung fibroblast cells and normal human lung tissue were re-analyzed using the GenePattern software program (http://www.broadinstitute.org) as described in detail elsewhere (13).

Cell culture and proliferation assays. All cell lines were purchased from the American Type Culture Collection (ATCC) and cultured in RPMI-1640 (Invitrogen) with 5\% FBS (Hyclone), at $37^{\circ} \mathrm{C}$ in $10 \% \mathrm{CO}_{2}$. For drug treatments, 3,0005,000 cells/well, dependent on the cell lines, were seeded in 96-well plates and incubated overnight. Cells were then treated with either DMSO (control) or ponatinib at various concentrations. Cell viability was determined using the CellTiter-Glo ${ }^{\circledR}$ luminescence cell viability kits (Promega) and a SpectraMax ${ }^{\circledR}$ M5e (Molecular Probes) luminescence plate reader.

Cell apoptosis assay and cell cycle analysis. For analysis of apoptosis, cells were plated in 6-well plates and treated with ponatinib for $72 \mathrm{~h}$. The cells were then washed with PBS, stained with Annexin V-APC and 7-AAD (BD Biosciences) following the manufacturer's instructions. Appearance of Annexin V and 7-AAD in the flow cytometric analysis indicated onset of apoptosis. Cell cycle analysis was performed using standard flow cytometric procedures following either propidium iodide staining alone, or together with bromodeoxyuridine (BrdU) incorporation. These cells were then stained with anti-BrdUAPC (eBioscience), as described previously (14). Cells were analyzed using an LSRII flow cytometer. All flow cytometric data were analyzed using FlowJo software (Tree Star, Inc., Ashland, OR, USA).

Western blot analyses. Proteins were isolated using standard procedures. Whole-cell lysates containing $50 \mu \mathrm{g}$ of proteins, were separated by SDS-PAGE and immunoblotted with the following specific antibodies: anti-FGFR1 and GAPDH (Santa Cruz Biotechnology, Inc., Santa Cruz, CA, USA), anti-phosphoFGFR1 (Abcam), anti-Src and anti-pSrc (Cell Signaling Technologies, Danvers, MA, USA) and $\beta$-actin (Sigma, St. Louis, MO, USA) using standard protocols as described previously (15).

Immunocytochemical staining. Immunocytochemical analysis was performed as described previously (16). Briefly, the cells were grown on sterile glass coated coverslips, and treated with or without $1.0 \mu \mathrm{M}$ ponatinib for $1 \mathrm{~h}$, fixed and permeablized. Cells were first blocked with $3 \%$ ovalbumin for $30 \mathrm{~min}$ at $25^{\circ} \mathrm{C}$. They were then stained with 1:50 primary rabbit polyclonal anti-phospho-FGFR1 (Y653/654; Abcam) antibody at $4^{\circ} \mathrm{C}$ overnight. The cells were then washed with PBS and incubated further with the Cy2-conjugated goat anti-rabbit IgG (Abcam) secondary antibody $(1: 200)$ for $30 \mathrm{~min}$ at $37^{\circ} \mathrm{C}$. After two more washings, the slides were counterstained with Hoechst 33342 DNA dye before mounting. The images were captured using a Zeiss fluorescent microscope.

Molecular analyses. Total RNA was isolated using TRIzol (Invitrogen) reagent, and digested with RNA-free DNase to eliminate genomic DNA contamination. Reverse-transcription was achieved using a SuperScript first-strand synthesis system (Invitrogen), and amplified by PCR or quantitated by real-time RT-PCR using conditions described previously (13).

Clonogenicity assay. Cells (500-1,000/well) were seeded in 6-well plates, allowed to attach overnight to the plastic substrate before treatment with ponatinib $(1.0 \mu \mathrm{M})$ or vehicles (controls). After $48 \mathrm{~h}$ treatment, the media were replaced with drug-free media for the desired length of time. As the colonies became visible (usually 2-3 weeks), cells were fixed with methanol, stained with Giemsa (1:10 in distilled water), and counted. 
A

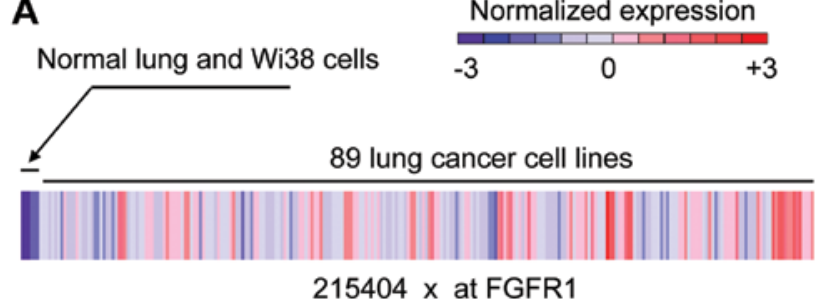

B

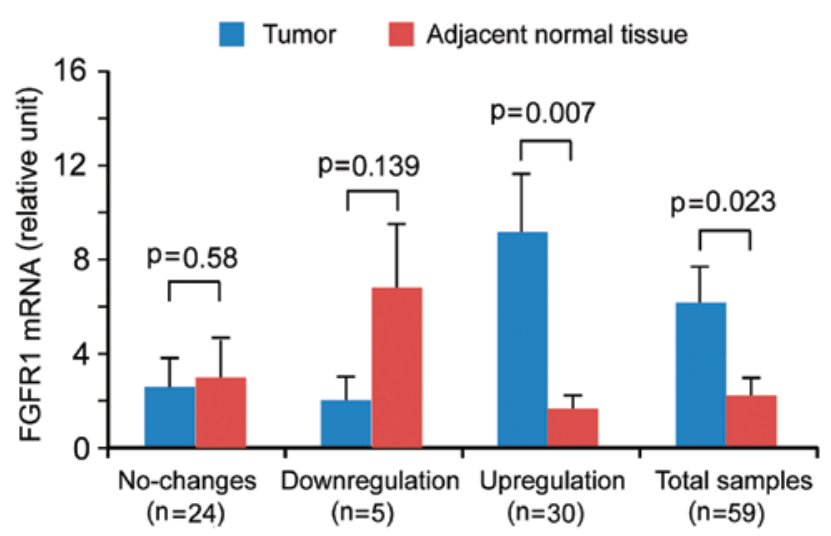

Figure 1. Overexpression of FGFR1 is observed in a proportion of NSCLC cell lines and tumor samples. (A) Analysis of published microarray data (E-MTAB-37) of lung cancer cell lines ( $\mathrm{n}=89$ ). Heatmap showed the upregulation of FGFR1 gene expression in individual lung cancer cell lines. (B) Real-time RT-PCR analysis revealed that $\sim 50 \%$ of surgically resected NSCLC specimens expressed markedly higher levels of FGFR1 mRNA when compared with their adjacent normal lung tissues.

shRNA knockdown of FGFRl. Retroviral shRNAs for FGFR1 were purchased from Thermo Scientific (catalog nos. RHS3979-9568791, 3979-98488862 and 3979-9568788, targeting different exons of FGFR1). shRNA-GFP was used as a control. Retroviral supernatants $\left(\geq 5 \times 10^{6} \mathrm{CFU} / \mu \mathrm{l}\right)$ were generated and introduced into cells as described previously (17), and puromycin was used for selection of clones where needed.

Statistical analysis. Data from the cell proliferation assay and clonogenicity assay are presented as means $\pm \mathrm{SD}$. Differences between groups were analyzed using the Student's t-test for independent samples. The level of significance was set at $\mathrm{p}<0.05$.

\section{Results}

Transcriptional overexpression of FGFRI is frequently observed in both lung cancer cell lines and human NSCLC tumor samples. To initiate the study, we first evaluated published global gene expression array datasets from the transcriptomics of cancer cell line project (950 assays, E-MTAB-37, European Bioinformatics Institute). This dataset contains triplicate expression profiles of various cancer cell lines. Using the GenePattern software program (http://www. broadinstitute.org) we re-analyzed data for lung cancer cell lines and found that FGFR1 was overexpressed in 38\% (35 out of 89) of lung cancer cell lines compared with Wi38 normal human fetal lung fibroblast cells and normal human lung tissue (Fig. 1A). To determine whether overexpression of FGFR1 also occurs in primary human lung cancers, we identified 88 NSCLC samples with matched normal lung tissues resected from lung cancer patients at the Shanghai Pulmonary
Hospital. After total RNA isolation, we identified 59 pairs of high quality matched RNA samples suitable for further analysis. The clinical features of these 59 NSCLC patients are presented in Table I. We used real-time RT-PCR to quantify human FGFR1 mRNA levels and demonstrated that 30 out of $59(50.8 \%)$ tumors showed a $>2$-fold increase in FGFR1 mRNA expression compared with their adjacent normal counterparts (Fig. 1B). Five (8.5\%) tumors showed lower expression levels compared with the matched normal lung tissues (Fig. 1B). We did not observe a significant difference between smokers and non-smokers (Table I).

Ponatinib effectively inhibits cell growth and colony formation in lung cancer cell lines overexpressing FGFR1. The frequent upregulation of FGFR in primary lung cancers suggests that targeting the FGFR1 signaling pathway using pharmacological inhibitors may be an effective way to restrict NSCLC growth. Our previous research targeting FGRF1 fusion kinases in stem cell leukemia/lymphoma syndrome, demonstrated that ponatinib, a multiple tyrosine kinase inhibitor, can effectively suppress FGFR1 fusion kinase activity and subsequently inhibit cell growth in vitro and leukemogenesis in mouse models in vivo (12). This study demonstrated that ponatinib was more potent than several other available FGFR1 inhibitors, such as TKI258 and PD17142. To determine whether this was also the case for NSCLC, we analyzed FGFR1 expression levels in 12 NSCLC cell lines using RT-PCR. Three of these cell lines H1299, A549 and H520, showed FGFR1 overexpression compared to $\mathrm{H} 3122$, which was confirmed using western blot analysis (Fig. 2A). H520 is a squamous cell line that carries FGFR1 amplification (18). H1299 and A549 (both 
Table I. Clinical characteristics of patients.

\begin{tabular}{|c|c|c|c|c|c|c|}
\hline No. of specimen & Gender & Age & Smoker & TNM stage & Location, histology, differentiation & FGFR1 level \\
\hline 9 & M & 67 & $\mathrm{~N}$ & $\mathrm{Ib}$ & LLL, SQC, moderate & Down \\
\hline 10 & M & 74 & $\mathrm{Y}$ & Ia & RUL, SQC, moderate & UNC \\
\hline 11 & M & 57 & $\mathrm{Y}$ & IIIa & RLL, SQC, moderate & UNC \\
\hline 14 & M & 59 & $\mathrm{~N}$ & $\mathrm{Ib}$ & LLL, ADC, well & UNC \\
\hline 15 & M & 53 & $\mathrm{Y}$ & IIIa & RML, SQC, moderate & Down \\
\hline 16 & M & 57 & $\mathrm{~N}$ & IIIA & RLL, ADC, poor to moderate & UNC \\
\hline 18 & M & 55 & $\mathrm{Y}$ & $\mathrm{IIb}$ & RUL, ADC, well & UNC \\
\hline 20 & M & 55 & $\mathrm{Y}$ & $\mathrm{Ib}$ & LLL, SQC, moderate & UNC \\
\hline 21 & M & 58 & $\mathrm{Y}$ & IIa & LLL, SQC, moderate & UNC \\
\hline 22 & M & 72 & $\mathrm{Y}$ & $\mathrm{Ib}$ & RUL, SQC & $\mathrm{Up}$ \\
\hline 23 & M & 71 & $\mathrm{~N}$ & $\mathrm{Ib}$ & RUL, ADC, moderate to well & UNC \\
\hline 26 & $\mathrm{~F}$ & 55 & $\mathrm{~N}$ & $\mathrm{IIb}$ & LLL, ADC, moderate & Up \\
\hline 27 & M & 74 & $\mathrm{Y}$ & $\mathrm{Ib}$ & LUL, SQC, well & Up \\
\hline 28 & M & 75 & $\mathrm{Y}$ & $\mathrm{IIb}$ & RUL, SQC, poor & UNC \\
\hline 29 & $\mathrm{~F}$ & 57 & $\mathrm{~N}$ & $\mathrm{Ib}$ & RLL, ADC, moderate & $\mathrm{Up}$ \\
\hline 30 & M & 78 & $\mathrm{~N}$ & IIb & RLL, ADC, well & UNC \\
\hline 31 & $\mathrm{~F}$ & 74 & $\mathrm{~N}$ & $\mathrm{Ib}$ & RML, ADC, well & Down \\
\hline 32 & M & 59 & $\mathrm{~N}$ & $\mathrm{Ib}$ & RUL, ADC, poor to moderate & $\mathrm{Up}$ \\
\hline 34 & M & 59 & $\mathrm{~N}$ & IIIb & LUL, ADC & $\mathrm{Up}$ \\
\hline 35 & $\mathrm{~F}$ & 63 & $\mathrm{~N}$ & $\mathrm{Ib}$ & LUL, adenosquamous & UNC \\
\hline 37 & M & 65 & $\mathrm{Y}$ & IIIa & LLL, ADC, poor to moderate & Up \\
\hline 38 & M & 47 & $\mathrm{~N}$ & N/A & LUL, TB & $\mathrm{Up}$ \\
\hline 39 & M & 67 & $\mathrm{Y}$ & IIIb & LUL, SQC, well & Up \\
\hline 40 & M & 63 & $\mathrm{Y}$ & $\mathrm{Ib}$ & LUL, ADC, poor to moderate & Up \\
\hline 49 & $\mathrm{~F}$ & 63 & $\mathrm{~N}$ & IIIa & RUL, ADC, moderate & Down \\
\hline 51 & M & 77 & $\mathrm{Y}$ & IIIa & LLL, ADC, moderate to well & $\mathrm{Up}$ \\
\hline 52 & M & 74 & $\mathrm{Y}$ & IIIa & RML, RLL, adenosquamous & UNC \\
\hline 58 & M & 45 & $\mathrm{Y}$ & $\mathrm{IIb}$ & LUL, ADC, partially mucinous & UNC \\
\hline 59 & M & 59 & $\mathrm{~N}$ & IIIa & RUL, ADC, poor to moderate & UNC \\
\hline 61 & $\mathrm{~F}$ & 62 & $\mathrm{~N}$ & IIIa & RLL, ADC, poor to moderate & $\mathrm{Up}$ \\
\hline 62 & M & 71 & $\mathrm{Y}$ & IIIb & LUL, AQC, moderate & UNC \\
\hline 63 & $\mathrm{~F}$ & 66 & $\mathrm{~N}$ & $\mathrm{Ib}$ & RLL, mucinous & $\mathrm{Up}$ \\
\hline 64 & $\mathrm{~F}$ & 38 & $\mathrm{~N}$ & IIb & LLL, mucinous with bronchioalveolar & UNC \\
\hline 65 & M & 69 & $\mathrm{Y}$ & IIIa & RML, RLL, SQC, moderate to well & $\mathrm{Up}$ \\
\hline 66 & M & 63 & $\mathrm{~N}$ & $\mathrm{Ib}$ & LUL, SQC, poor to moderate & UNC \\
\hline 67 & M & 73 & $\mathrm{Y}$ & $\mathrm{Ib}$ & LUL, adenosquamous & $\mathrm{Up}$ \\
\hline 68 & $\mathrm{~F}$ & 64 & $\mathrm{~N}$ & $\mathrm{Ib}$ & RUL, sarcoma & $\mathrm{Up}$ \\
\hline 69 & M & 68 & $\mathrm{Y}$ & IIb & LUL, SQC, moderate & $\mathrm{Up}$ \\
\hline 74 & M & 63 & $\mathrm{Y}$ & N/A & RML, nasopharyngeal SQC & Up \\
\hline 75 & M & 49 & $\mathrm{Y}$ & IIa & LLL, ADC, poor to moderate & $\mathrm{Up}$ \\
\hline 76 & M & 53 & $\mathrm{~N}$ & $\mathrm{IIb}$ & RLL, ADC, poor to moderate & $\mathrm{Up}$ \\
\hline 77 & $\mathrm{~F}$ & 54 & $\mathrm{~N}$ & $\mathrm{IIb}$ & LUL, SQC, moderate & $\mathrm{Up}$ \\
\hline 78 & M & 78 & $\mathrm{~N}$ & $\mathrm{Ib}$ & RLL, SQC, poor & Up \\
\hline 79 & $\mathrm{~F}$ & 52 & $\mathrm{~N}$ & IIIa & RML, large cell & $\mathrm{Up}$ \\
\hline 81 & M & 62 & $\mathrm{~N}$ & N/A & RUL, TB & UNC \\
\hline 82 & $\mathrm{~F}$ & 75 & $\mathrm{Y}$ & IIIa & LUL, SQC, moderate & UNC \\
\hline 83 & M & 45 & $\mathrm{Y}$ & IIIb & LUL, SQC, moderate & $\mathrm{Up}$ \\
\hline 84 & M & 59 & $\mathrm{Y}$ & IV & RLL, atypical carcinoid & $\mathrm{Up}$ \\
\hline 85 & $\mathrm{~F}$ & 38 & $\mathrm{~N}$ & N/A & Mediastinal mass, lymphoma & UNC \\
\hline 86 & M & 61 & $\mathrm{Y}$ & IIIa & RLL, SQC, poor to moderate & UNC \\
\hline 87 & M & 63 & $\mathrm{Y}$ & $\mathrm{Ib}$ & LLL, ADC, moderate to well & $\mathrm{Up}$ \\
\hline 88 & M & 78 & $\mathrm{~N}$ & $\mathrm{Ib}$ & LLL, SQC, moderate & UNC \\
\hline 89 & $\mathrm{~F}$ & 53 & $\mathrm{~N}$ & $\mathrm{Ib}$ & LUL, SQC, moderate & $\mathrm{Up}$ \\
\hline 90 & $\mathrm{~F}$ & 59 & $\mathrm{~N}$ & N/A & RUL, TB & UNC \\
\hline 91 & M & 62 & $\mathrm{Y}$ & IIIa & RML, SQC, moderate & UNC \\
\hline 92 & M & 63 & $\mathrm{~N}$ & $\mathrm{Ib}$ & RLL, SQC, well & UNC \\
\hline 93 & M & 70 & $\mathrm{Y}$ & $\mathrm{Ib}$ & RML, sarcomatoid & Up \\
\hline 94 & M & 39 & $\mathrm{Y}$ & $\mathrm{Ib}$ & RML, SQC, poor to moderate & $\mathrm{Up}$ \\
\hline 97 & M & 63 & $\mathrm{~N}$ & IIIa & LUL, ADC, moderate to well & UNC \\
\hline
\end{tabular}

LUL, left upper lobe; LLL, left lower lobe; RUL, right upper lobe; RML, right middle lobe; RLL, right lower lobe; ADC, adenocarcinoma; SQC, squamous cell carcinoma; Up, upregulated; Down, downregulated; UNC, unchanged, N/A, not applicable; TB, tuberculosis. 
A

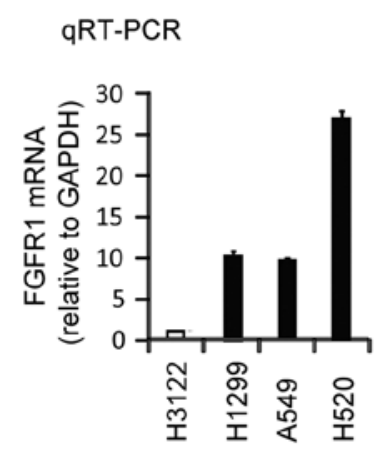

WB

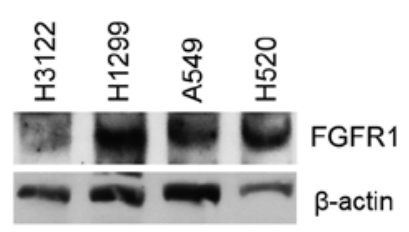

B

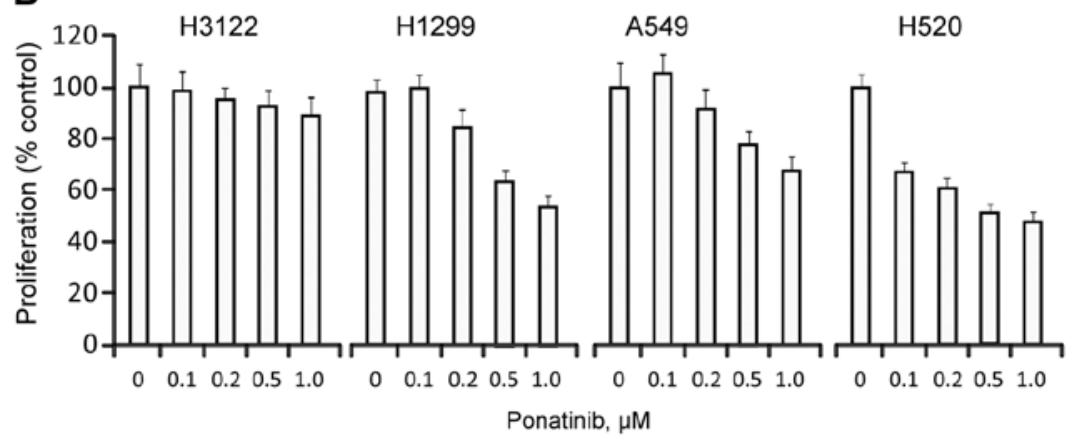

C

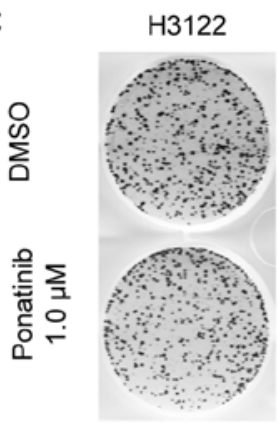

H1299

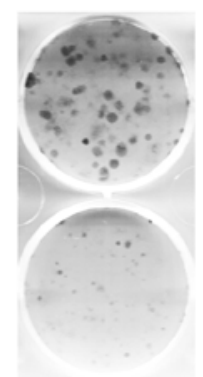

A549

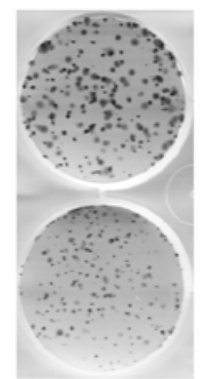

H520

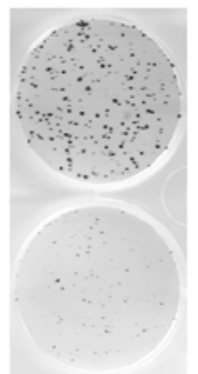

Figure 2. Inhibition of FGFR activation by ponatinib is associated with cell growth suppression. (A) Quantitative real-time RT-PCR (qRT-PCR) and western blot (WB) analysis of FGFR1 expression in NSCLC cell lines H1299, A549 (both undifferentiated) and H520 (squamous cell, FGFR1 amplified) compared to H3122 (adenocarcinoma, EML4-ALK translocation). The FGFR1 transcription level of H3122 was set as 1 and $\beta$-actin (ACTB) was used as loading control. (B) Effects of ponatinib on cell proliferation. Values are expressed as a percentage of the control (DMSO). Each value represents an average of three independent experiments. (C) Clonogenic assay revealed that the number of colonies was markedly decreased in the presence of $1.0 \mu \mathrm{M}$ of ponatinib when compared to those of the DMSO controls.

undifferentiated), and H3122 (adenocarcinoma, EML4-ALK fusion positive) do not carry FGFR1 amplification or mutations (Cancer Cell Line Encyclopedia, http://www.broadinstitute.org/ ccle/home). These cell lines were used to determine whether ponatinib affects cell growth using a range of concentrations. Ponatinib showed no effect on the growth of $\mathrm{H} 3122$ cells which express low levels of FGFR1 when used at concentrations up to $1000 \mathrm{nM}$ (Fig. 2B). In contrast, the three cell lines overexpressing FGFR1 showed a dramatic reduction in cell growth rate at various concentrations, ranging from 200 to $1000 \mathrm{nM}$. We further used a colony formation assay to evaluate the effect of ponatinib treatment. Similar to the cell proliferation effects, ponatinib significantly inhibited colony formation in cells overexpressing FGFR1 but not in H3122 cells which express low levels of FGFR1 (Fig. 2C).

Ponatinib suppresses activation of FGFRl and downstream targets and leads to inhibition of cell division. Having shown that ponatinib inhibits cell growth of NSCLC cells overexpressing FGFR1, we then determined whether this cell growth inhibition is associated with decreased levels of phospho-FGFR1. Ponatinib dramatically decreased the tyrosine phosphorylation levels in all three cell lines expressing FGFR1 (Fig. 3A). Western blot analyses with phospho-specific antibodies showed that ponatinib treatment also led to inactivation of several direct targets of FGFR1, such as Src and PLC $\gamma$ (Fig. 3B). Since ponatinib directly inhibits Src activation (10), the level of phospho-Src in H3122 was also diminished (Fig. 3A). Inactivation of Src, however, did not lead to growth inhibition of the H3122 cells (Fig. 2B). Consistently, immunocytochemical analysis using a specific anti-phospho-FGFR1 antibody further confirmed that ponatinib $(1.0 \mu \mathrm{M})$ effectively reduced the membrane phospho-FGFR1 staining levels in both H1299 and A549 cells (Fig. 3B) after only 1 h. In parallel with these analyses, we also determined whether downregulation of FGFR1 activation could lead to cell cycle arrest or/and cell apoptosis. Ponatinib markedly inhibited the cell cycle in these FGFR1-positive cells but not in FGFR1-negative H3122 cells (Fig. 3C). No dramatic increase in the apoptotic cell population was noted in the ponatinib-treated group (data not shown). To further investigate whether cell growth inhibition is related to inactivation of FGFR1 kinase by ponotinib, we treated the H520 and H1581 cells with ponatinib in step-wise increasing doses. The H1581 cell line has been shown to overexpress FGFR1 (18), FGFR2 and FGFR4 (Fig. 6). The H520 cells, however, mainly overexpress FGFR1 with only low levels of FGFR2-4 compared with the H1581 cells. Other NSCLC cell lines showed a similar expression pattern as the H520 cell line (Fig. 6). Immunoblotting, with the anti-phospho-FGFR1 antibody, demonstrated that the FGFR1 activity in the H1581 cells was inhibited by a lower dose of ponatinib than that used for the H520 cell line (Fig. 3D). Consistently, the cell proliferation assay showed that the H1581 cells were more sensitive to ponatinib when compared with the $\mathrm{H} 520$ cells. The $\mathrm{IC}_{50}$ values for the H1581 and $\mathrm{H} 520$ cells were 30 and $50 \mathrm{nM}$, respectively (Fig. 3E). Collectively, these data suggest that cell growth inhibition by ponatinib correlates with inhibition of FGFR1 activation. 

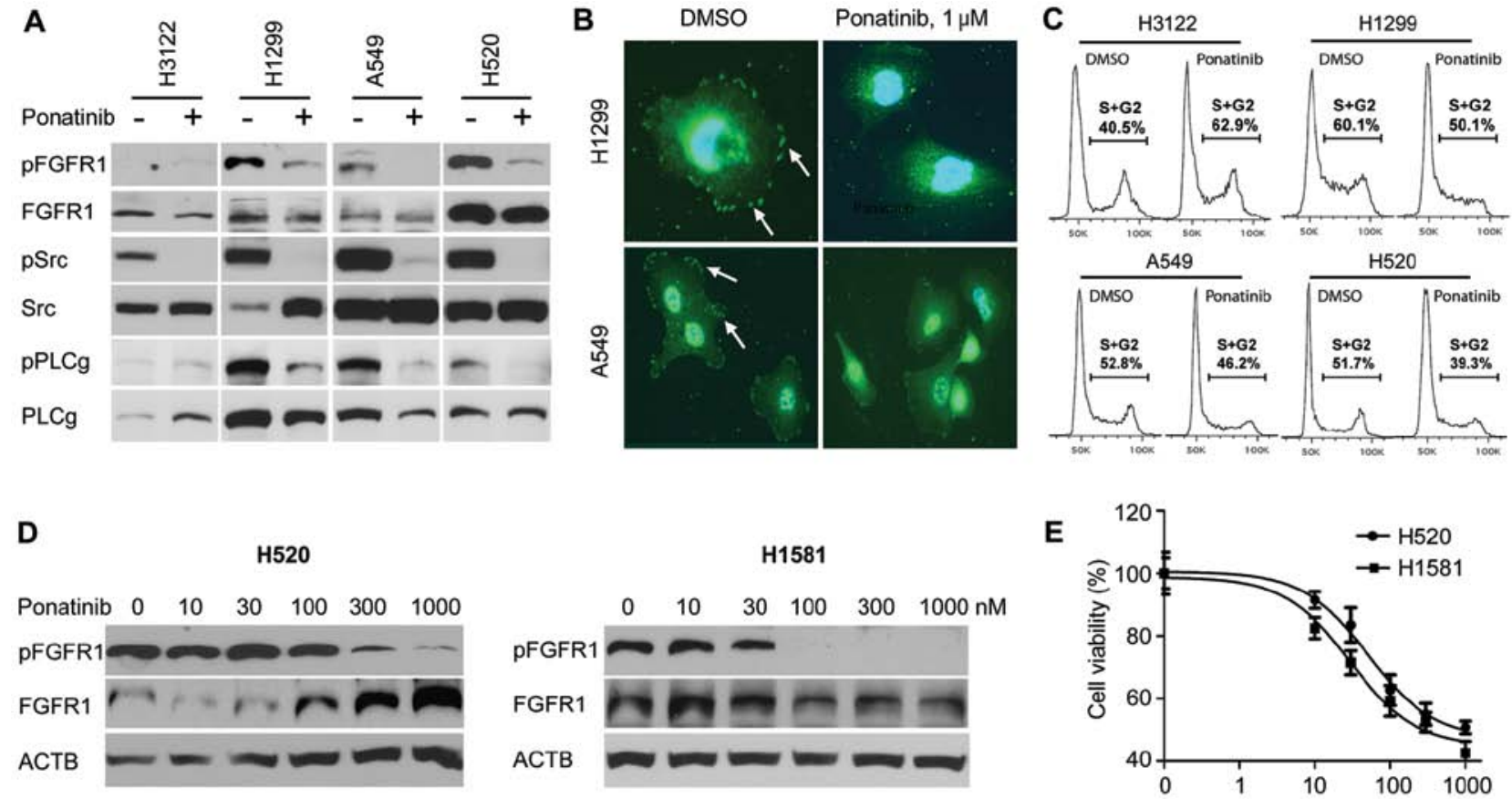

Figure 3. Downregulation of FGFR1 activation by ponatinib results in cell growth inhibition. (A) Western blot analysis of phospho-FGFR1 levels in four NSCLC cell lines using the anti-pY654 antibody demonstrated that ponatinib treatment $(1.0 \mu \mathrm{M})$ led to reduced levels of phospho-FGFR1 while it did not affect total FGFR1. Inhibition of FGFR1 activation by ponatinib $(1.0 \mu \mathrm{M})$ also resulted in reduced activation of the FGFR1 downstream effectors Src and PLC $\gamma$. (B) Immunocytochemical analysis revealed that the density of membrane-bound phospho-FGFR1 staining (Y653/654, arrows) was reduced by ponatinib treatment at $1.0 \mu \mathrm{M}$. (C) Ponatinib caused accumulation of cells in the G1 phase in NSCLC cells overexpressing FGFR1. (D) Western blot analysis indicating the phospho-FGFR1 levels in the H1581 and H520 cells treated with ponatinib for $24 \mathrm{~h}$. (E) H1581 and H520 cells were incubated with ponatinib for $48 \mathrm{~h}$, and cell growth inhibition was assessed. Error bars refer to the SD of octuplicate analyses.

Knockdown of FGFR1 expression induces cell growth inhibition in NSCLC cell lines overexpressing FGFR1. Ponatinib was designed to act against BCR-ABL kinase and its mutants, but it also effectively inhibits FGFR1, SRC, VEGFR, PDGFR and KIT (10). To determine whether the growth inhibition noted in the three NSCLC cell lines was the direct result of inactivation of FGFR1, we used shRNAs to specifically knockdown FGFR1 expression in these cells. shGFP was used as a negative control. Seventy-two hours post transfection, cells from independently transfected wells were pooled for analysis. The pooled cells were coincidently analyzed for FGFR1 expression, activation, cell cycle analysis and cell proliferation. Real-time RT-PCR analysis showed that shFGFR1 markedly decreased the transcription levels of FGFR1 in all three NSCLC cell lines compared with the vector control carrying shGFP. The FGFR1-negative H3122 cells showed no alterations in expression as predicted (Fig. 4A). Consistently, western blotting with an anti-FGFR1 antibody also showed the effect of the knockdown in the shFGFR1 but not in the shGFP-infected cells (Fig. 4B). To determine whether knockdown of FGFR1 can decrease cell proliferation, we performed a standard cell cycle analysis which showed that knockdown of FGFR1 dramatically decreased the percentage of S/G2 phase cells compared with that in the shGFP-infected cells (Fig. 4C). Consistent with the cell cycle analysis, cell proliferation assay showed that shFGFR1 transduction significantly induced cell growth inhibition in the FGFR1-positive NSCLC cells compared with that in the shGFP-infected cells. We did not observe any differences between the two groups in the FGFR1-negative H3122 cells (Fig. 4D).
Ponatinib effectively inhibits human primary lung cancer cell culture growth. To investigate whether ponatinib can decrease the growth of primary lung cancer cells, we seeded freshly resected human non-small cell lung cancers into culture from 12 patients. Two of these cultures were successfully established and assayed. One was derived from a squamous-cell carcinoma ( $\mathrm{Pt} \mathrm{\# 8)}$ and another was from an adenocarcinoma (Pt \#9) based on their histological features (Fig. 5A, left panel). These two primary lung cancer cell cultures were routinely grown in RPMI-1640 medium plus 10\% FBS without addition of any growth factors. In the absence of ponatinib these cells overexpressed many oncogenes frequently upregulated in cancer e.g. Plk1, survivin and RSK2, compared to normal lung tissue (data not shown). Western blot analysis with FGFR1 antibodies showed high levels of expression of FGFR1 in these two tumor cell cultures compared to normal lung tissues and HBE4 cells (an immortalized human bronchial epithelial cell line) (Fig. 5A, right panel). Consistently, both primary cell cultures were sensitive to ponatinib treatment and showed a dramatic reduction in the cell growth rate at various $\mathrm{IC}_{50}$ values, ranging from 0.5 to $1.0 \mu \mathrm{M}$ (Fig. 5B). Further analysis suggested that this cell growth inhibition was associated with a decrease in phospho-FGFR1 levels in the presence of ponatinib (Fig. 5A, right bottom panel). To confirm the cell proliferation effect of ponatinib, we also performed colony formation assays, which demonstrated that ponatinib significantly inhibited colony formation of both primary lung cancer cell cultures (Fig. 5C). Subsequently, cell cycle analysis, in combination with BrdU uptake, showed that the percentage of cells in the $\mathrm{S}$ phase was dramatically decreased. Apoptotic 

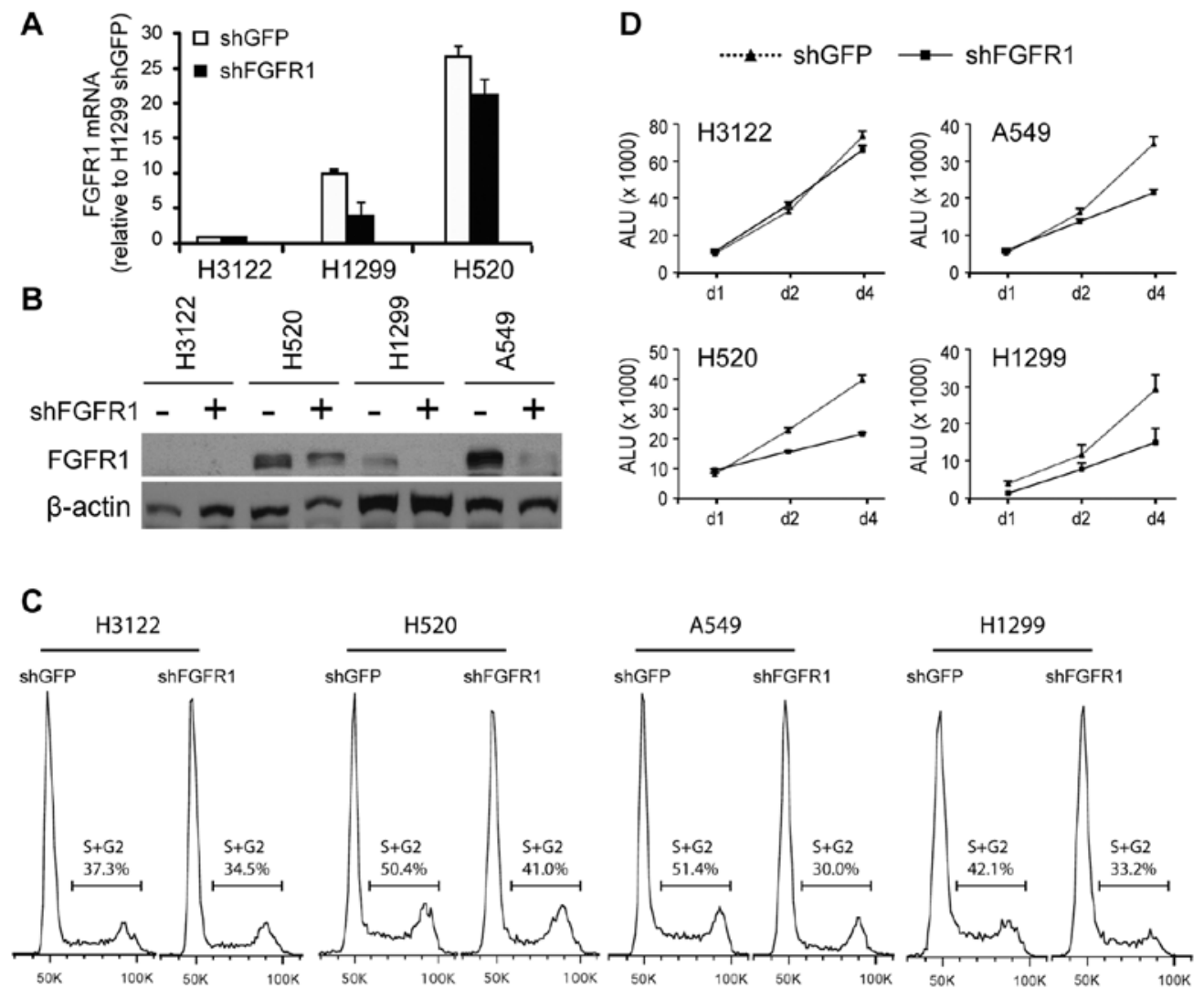

Figure 4. Knockdown of FGFR1 expression induces cell growth inhibition in NSCLC cell lines overexpressing FGFR1. Transient knockdown of FGFR1 using shRNAs resulted in the downregulation of (A) FGFR1 transcription and (B) FGFR1 protein expression. shFGFR1 caused accumulation of cells in (C) the G1 phase and (D) inhibited cell growth. ALU, arbitrary luminescence unit.

cells were not observed (Fig. 5D). These results indicate that ponatinib has a profound effect on human lung cancer cells expressing increased levels of FGFR1.

\section{Discussion}

Cancer is driven by different types of genetic mutations acquired during tumorigenesis and tumor growth (19). Identifying these mutations that are critical for tumor growth (oncogenic drivers) is the first step. Once the oncogenic drivers are identified, they can then be targeted accordingly. This therapeutic strategy is becoming increasingly more possible due to advances in second generation sequencing technology and other profiling platforms (20) which facilitate the identification of driver mutations together with the increasing availability of drugs that target these molecular changes (21). To date, EGFR $(22,23)$, KRAS (24), EML4-ALK (25), ROS1 (26,27), RET $(28,29)$, ERBB2 and PIK3CA (30) and MET (31), among others, are found to drive tumorigenesis in varying proportions of NSCLC, mostly adenocarcinomas. Targeted therapies have materialized in these tumors carrying EGFR, EML4-ALK, ROS1 as well as RET. In the case of FGFR1, however, there is no mutation(s) or translocation found to be involved, to date. Instead, copy number increase appears to be responsible for the activation of this pathway particularly in the squamous cell subtype $(18,32)$. Other important mechanisms for over-activation of FGFR1 may be caused by increasing availability of FGFR1 through increased transcription and/or autocrine/paracrine mechanisms as part of the epithelial to mesenchymal transition (EMT) (33). In the present study, we showed that $50 \%$ (30 out of 59) of the NSCLC patients overexpressed FGFR1, a percentage that is much higher than that of FGFR1 amplification. Together these studies indicate that i) copy number increase of FGFR1 contributes to the overexpression of FGFR1 in these tumor cells and ii) overexpression of FGFR1 in a proportion of patients is due to transcriptional upregulation of FGFR1. Given that aberrant FGFR pathway activation is also involved in drug resistance (34), FGFR targeting is an important strategy in containing cancer growth and possibly in overcoming resistance. Importantly, in two previous studies, while the FGFR1 inhibitor PD173074 was shown to be effective against cells with overactivated FGFR1, this was not uniformly effective suggesting other mutation(s) may affect sensitivity to PD173074.

In the present study, we demonstrated that targeting the FGFR1 signaling pathway using a novel FGFR1 inhibitor in NSCLC cell lines as well as primary NSCLC samples overexpressing FGFR1 significantly inhibited cancer cell growth and clonogenicity, suggesting the potential clinical benefit of ponatinib. It is noteworthy that two NSCLC cell lines, H1581 and H520, both harboring the FGFR1 gene amplification [4 copies (18)], have much higher levels of FGFR1 transcription than other cell lines (Figs. 2A). Consequently these two cell lines were more sensitive to ponatinib (Fig. 3D and E; $\mathrm{IC}_{50}<50 \mathrm{nM}$ ), confirming the results from a recent study (35). At the recommended clinical trial dose of $45 \mathrm{mg}$, the trough level of ponatinib reached $40 \mathrm{nM}$. The peak level was several 

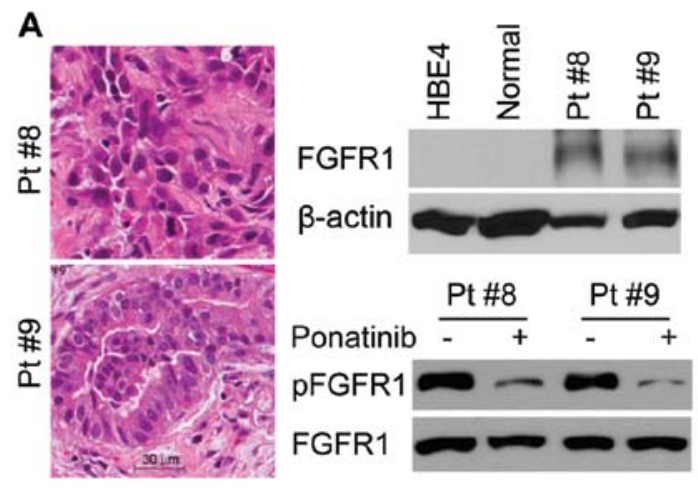

B
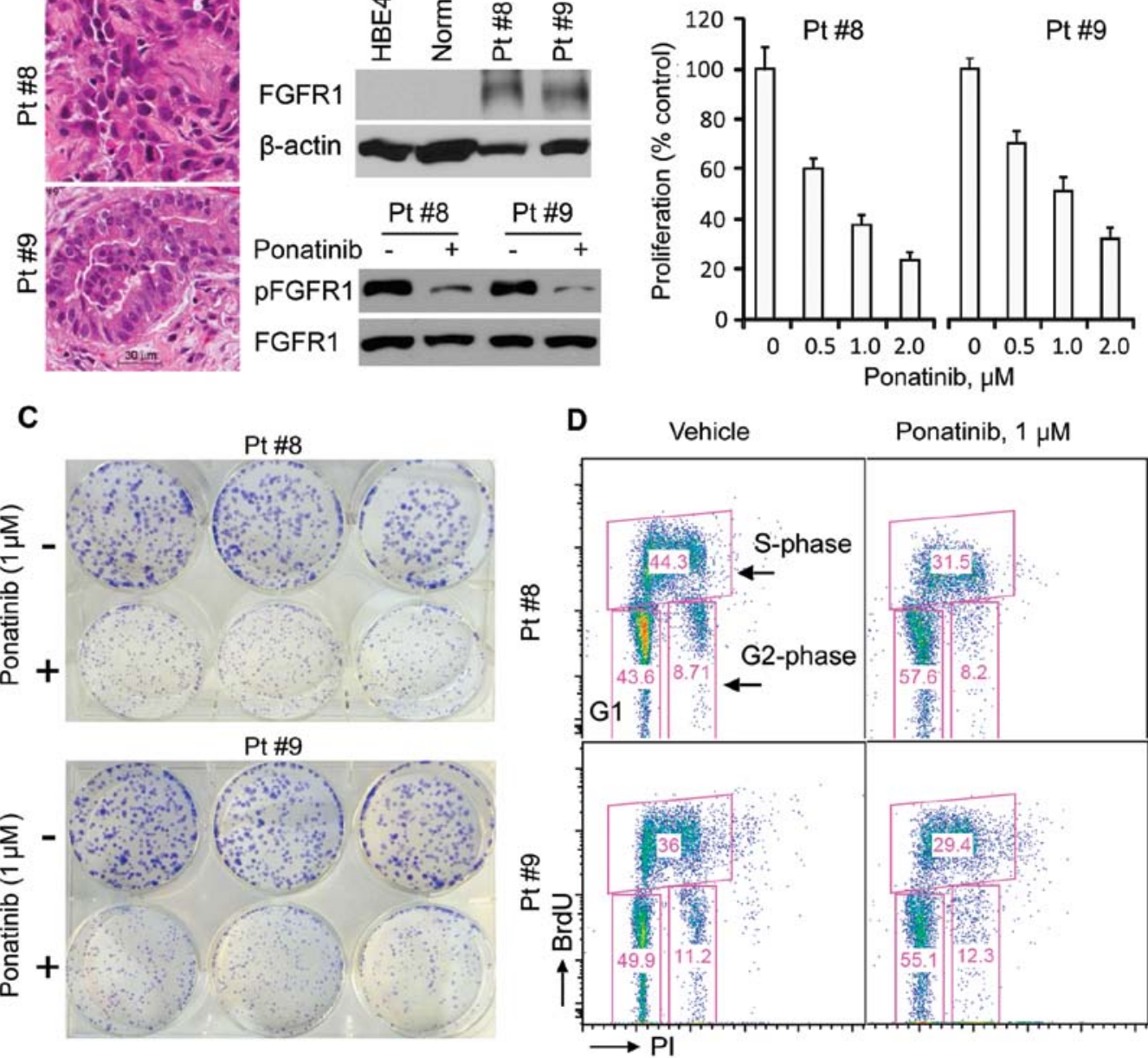

Figure 5. Ponatinib treatment effectively inhibits human primary lung cancer cell culture growth. (A) Histological studies of two primary lung cancer tissues (left panel) and western blot analysis (right panel) showed high expression of FGFR1 in the two primary lung cancer tissues when compared with a normal human lung tissue and HBE4 immortalized human bronchial epithelial cells (right top panel). The phospho-FGFR1 levels in the primary lung cell cultures were significantly reduced following ponatinib treatment at $1.0 \mu \mathrm{M}$ (right bottom panel). (B) Cell proliferation assays showed that ponatinib inhibited the growth of the primary lung cell cultures at various concentrations. Error bars refer to the SD of octuplicate analyses. (C) Clonogenic assay showed that the number of colonies was decreased in the presence of ponatinib at $1.0 \mu \mathrm{M}$. (D) BrdU cell cycle analysis showed a decrease in the percentage of cells in the $\mathrm{S}$ phase following treatment with $1.0 \mu \mathrm{M}$ ponatinib. All experiments were performed at least in duplicate.

A

FGFR1

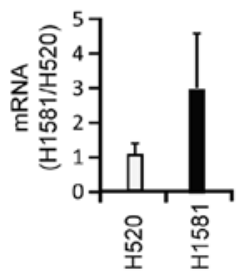

B

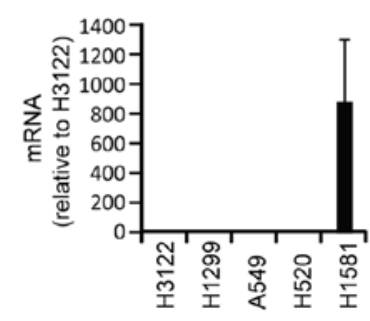

FGFR3

FGFR4

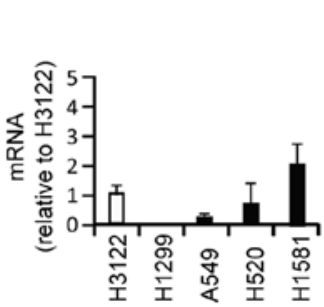

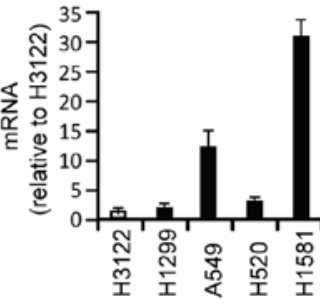

Figure 6. Relative gene expression levels of all FGFR isoforms in lung cancer cell lines. Real-time RT-PCR analysis elucidated (A) the relative FGFR1 transcription level of H1581 versus H520, and (B) the transcriptional levels of FGFR2-4 from five NSCLC cell lines. Data are presented as means \pm SD from triplicate measurements.

fold higher (35). This suggests that ponatinib at a clinically achievable concentration markedly reduces the growth of NSCLC in which FGFR1 is amplified. However, further studies in animal models are necessary in order to determine the efficacy before clinical trials are initiated in lung cancer patients whose tumors overexpress FGFR1.
It is noteworthy that an increasing number of studies have demonstrated that overexpression of FGFR1 is not only observed in NSCLC, but also in other types of cancers, such as breast cancer (36-38), prostate cancer $(39,40)$ and ovarian cancer (41). Recent large scale analyses of somatic copynumber alterations from 3,131 cancer specimens, spanning 
26 different cancer types, found amplification of the FGFR1 gene in $10 \%$ of cases. This is the most significantly reported focal amplification (42), indicating that FGFR1 signaling is commonly involved in tumorigenesis and/or tumor progression and may represent a promising therapeutic target in many cancer types. The precise role of FGFR1 signaling in the pathogenesis and progression of these tumors, however, is still unclear. In rearranged FGFR1 fusion kinase-induced leukemia and lymphoma, we and others $(17,43)$ have demonstrated that constitutive activation of the chimeric FGFR1 kinase alone is not sufficient to cause overt leukemia/lymphoma development in mouse models. Using immunohistochemical staining, Behrens et al (4) studied 321 NSCLC tissue samples and 426 adjacent bronchial epithelial specimens, and found a significant increase in bFGF and FGFR proteins in respiratory epithelium with squamous dysplasia, compared with metaplastic bronchial epithelia. Using SNP array analysis, Dutt et al (32) also observed that 60\% (11 out of 19) of primary NSCLC patients were in stage I. Together these observations suggest that the activation of the FGFR signaling pathway is an early event in the pathogenesis of NSCLC. If so, FGFR inhibitors may prove to be effective against early stage tumors.

In summary, our study provides convincing evidence that abnormal expression of FGFR1 occurs in almost $50 \%$ of all NSCLC patients presented with both major histological types. FGFR1, therefore, may serve as a novel target in molecularly targeted therapies against lung cancer. Targeting FGFR1 kinases with the novel FGFR1 inhibitor ponatinib is a potentially successful approach for the treatment of NSCLC patients carrying aberrant activation of FGFR1. It is conceivable that ponatinib may be used where appropriate either alone or in combination with other drugs in the future.

\section{Acknowledgements}

We thank Scott Antonia of the Lee Moffitt Cancer Center and Matthew Meyerson of Dana-Farber Cancer Institute, Harvard Medical School, for sharing their non-small cell cancer cell lines. We are grateful to Ms. Haiyan Qin for technical support in the molecular analyses and Michael Boyd for assistance with micrograph preparation from the pathology slides. Ponatinib was kindly provided by Ariad Pharmaceuticals, Inc.

\section{References}

1. Stinchcombe TE, Bogart J, Wigle DA and Govindan R: Annual review of advances in lung cancer clinical research: a report for the year 2009. J Thorac Oncol 5: 935-939, 2010.

2. Perez-Moreno P, Brambilla E, Thomas R and Soria JC: Squamous cell carcinoma of the lung: molecular subtypes and therapeutic opportunities. Clin Cancer Res 18: 2443-2451, 2012.

3. Subramanian J, Corrales L, Soulieres D, Morgensztern D and Govindan R: Summary of presentations from the 46th Annual Meeting of the American Society of Clinical Oncology (2010) focus on tumor biology and biomarkers related to lung cancer. $\mathrm{J}$ Thorac Oncol 6: 399-403, 2011.

4. Behrens C, Lin HY, Lee JJ, et al: Immunohistochemical expression of basic fibroblast growth factor and fibroblast growth factor receptors 1 and 2 in the pathogenesis of lung cancer. Clin Cancer Res 14: 6014-6022, 2008.

5. Sasaki H, Shitara M, Yokota K, et al: Increased FGFR1 copy number in lung squamous cell carcinomas. Mol Med Rep 5: 725-728, 2012.

6. Peifer M, Fernandez-Cuesta L, Sos ML, et al: Integrative genome analyses identify key somatic driver mutations of small-cell lung cancer. Nat Genet 44: 1104-1110, 2012.
7. Turner $\mathrm{N}$ and Grose R: Fibroblast growth factor signalling: from development to cancer. Nat Rev Cancer 10: 116-129, 2010.

8. Larsson H, Klint P, Landgren E and Claesson-Welsh L: Fibroblast grow th factor receptor-1-mediated endothelial cell proliferation is dependent on the Src homology (SH) 2/SH3 domain-containing adaptor protein Crk. J Biol Chem 274: 25726-25734, 1999.

9. Sandilands E, Akbarzadeh S, Vecchione A, McEwan DG, Frame MC and Heath JK: Src kinase modulates the activation, transport and signalling dynamics of fibroblast growth factor receptors. EMBO Rep 8: 1162-1169, 2007.

10. O'Hare T, Shakespeare WC, Zhu X, et al: AP24534, a pan-BCRABL inhibitor for chronic myeloid leukemia, potently inhibits the T315I mutant and overcomes mutation-based resistance. Cancer Cell 16: 401-412, 2009.

11. Chase A, Bryant C, Score J and Cross NC: Ponatinib as targeted therapy for FGFR1 fusions associated with the 8p11 myeloproliferative syndrome. Haematologica 98: 103-106, 2013.

12. Ren M, Qin H, Ren R and Cowell JK: Ponatinib suppresses the development of myeloid and lymphoid malignancies associated with FGFR1 abnormalities. Leukemia 27: 32-40, 2013.

13. Ren M and Cowell JK: Constitutive Notch pathway activation in murine ZMYM2-FGFR1-induced T-cell lymphomas associated with atypical myeloproliferative disease. Blood 117: 6837-6847, 2011.

14. Darzynkiewicz Z and Juan G: Analysis of DNA content and BrdU incorporation. Curr Protoc Cytom 7: unit 7.7, 2001. doi: 10.1002/0471142956.cy0707s02.

15. Ren M, Qin H, Ren R, Tidwell J and Cowell JK: Src activation plays an important key role in lymphomagenesis induced by FGFR1 fusion kinases. Cancer Res 71: 7312-7322, 2011.

16. Silva J, Wang G and Cowell JK: The temporal and spatial expression pattern of the LGI1 epilepsy predisposition gene during mouse embryonic cranial development. BMC Neurosci 12: 43, 2011.

17. Ren M, Li X and Cowell JK: Genetic fingerprinting of the development and progression of T-cell lymphoma in a murine model of atypical myeloproliferative disorder initiated by the ZNF198-fibroblast growth factor receptor-1 chimeric tyrosine kinase. Blood 114: 1576-1584, 2009.

18. Weiss J, Sos ML, Seidel D, et al: Frequent and focal FGFR1 amplification associates with therapeutically tractable FGFR1 dependency in squamous cell lung cancer. Sci Transl Med 2: 62ra93, 2010.

19. Stratton MR: Exploring the genomes of cancer cells: progress and promise. Science 331: 1553-1558, 2011.

20. Garnett MJ, Edelman EJ, Heidorn SJ, et al: Systematic identification of genomic markers of drug sensitivity in cancer cells. Nature 483: 570-575, 2012.

21. Ashworth A, Lord CJ and Reis-Filho JS: Genetic interactions in cancer progression and treatment. Cell 145: 30-38, 2011.

22. Mok TS, Wu YL, Thongprasert S, et al: Gefitinib or carboplatinpaclitaxel in pulmonary adenocarcinoma. N Engl J Med 361: 947-957, 2009.

23. Zhou C, Wu YL, Chen G, et al: Erlotinib versus chemotherapy as first-line treatment for patients with advanced EGFR mutationpositive non-small-cell lung cancer (OPTIMAL, CTONG-0802): a multicentre, open-label, randomised, phase III study. Lancet Oncol 12: 735-742, 2011.

24. Rosell R, Li S, Skacel Z, et al: Prognostic impact of mutated K-ras gene in surgically resected non-small cell lung cancer patients. Oncogene 8: 2407-2412, 1993.

25. Kwak EL, Bang YJ, Camidge DR, et al: Anaplastic lymphoma kinase inhibition in non-small-cell lung cancer. N Engl J Med 363: 1693-1703, 2010.

26. Bergethon K, Shaw AT, Ou SH, et al: ROS1 rearrangements define a unique molecular class of lung cancers. J Clin Oncol 30: 863-870, 2012.

27. Janne PA and Meyerson M: ROS1 rearrangements in lung cancer: a new genomic subset of lung adenocarcinoma. J Clin Oncol 30: 878-879, 2012.

28. Kohno T, Ichikawa H, Totoki Y, et al: KIF5B-RET fusions in lung adenocarcinoma. Nat Med 18: 375-377, 2012.

29. Takeuchi K, Soda M, Togashi Y, et al: RET, ROS1 and ALK fusions in lung cancer. Nat Med 18: 378-381, 2012.

30. Samuels Y and Velculescu VE: Oncogenic mutations of PIK3CA in human cancers. Cell Cycle 3: 1221-1224, 2004.

31. Kim ES and Salgia R: MET pathway as a therapeutic target. J Thorac Oncol 4: 444-447, 2009.

32. Dutt A, Ramos AH, Hammerman PS, et al: Inhibitor-sensitive FGFR1 amplification in human non-small cell lung cancer. PLoS One 6: e20351, 2011. 
33. Marek L, Ware KE, Fritzsche A, et al: Fibroblast growth factor (FGF) and FGF receptor-mediated autocrine signaling in nonsmall-cell lung cancer cells. Mol Pharmacol 75: 196-207, 2009.

34. Ware KE, Marshall ME, Heasley LR, et al: Rapidly acquired resistance to EGFR tyrosine kinase inhibitors in NSCLC cell lines through de-repression of FGFR2 and FGFR3 expression. PLoS One 5: e14117, 2010.

35. Gozgit JM, Wong MJ, Moran L, et al: Ponatinib (AP24534), a multi-targeted pan-FGFR inhibitor with activity in multiple FGFR-amplified or mutated cancer models. Mol Cancer Ther 11: 690-699, 2012.

36. Kwek SS, Roy R, Zhou H, et al: Co-amplified genes at $8 \mathrm{p} 12$ and 11 q13 in breast tumors cooperate with two major pathways in oncogenesis. Oncogene 28: 1892-1903, 2009.

37. Shiang CY, Qi Y, Wang B, et al: Amplification of fibroblast growth factor receptor-1 in breast cancer and the effects of brivanib alaninate. Breast Cancer Res Treat 123: 747-755, 2010.

38. Turner N, Pearson A, Sharpe R, et al: FGFR1 amplification drives endocrine therapy resistance and is a therapeutic target in breast cancer. Cancer Res 70: 2085-2094, 2010.
39. Freeman KW, Welm BE, Gangula RD, et al: Inducible prostate intraepithelial neoplasia with reversible hyperplasia in conditional FGFR1-expressing mice. Cancer Res 63: 8256-8263, 2003.

40. Freeman KW, Gangula RD, Welm BE, et al: Conditional activation of fibroblast growth factor receptor (FGFR) 1, but not FGFR2, in prostate cancer cells leads to increased osteopontin induction, extracellular signal-regulated kinase activation, and in vivo proliferation. Cancer Res 63: 6237-6243, 2003.

41. Mayr D, Kanitz V, Anderegg B, et al: Analysis of gene amplification and prognostic markers in ovarian cancer using comparative genomic hybridization for microarrays and immunohistochemical analysis for tissue microarrays. J Clin Pathol 126: 101-109, 2006.

42. Beroukhim R, Mermel CH, Porter D, et al: The landscape of somatic copy-number alteration across human cancers. Nature 463: 899-905, 2010.

43. Roumiantsev S, Krause DS, Neumann CA, et al: Distinct stem cell myeloproliferative/T lymphoma syndromes induced by ZNF198-FGFR1 and BCR-FGFR1 fusion genes from 8p11 translocations. Cancer Cell 5: 287-298, 2004. 